\title{
Low signal-to-noise spectroscopy and surface photometry of two faint galaxies in the field of NGC 7479
}

\author{
M. F. Saraiva ${ }^{1}$ and G. F. Benedict ${ }^{2}$ \\ ${ }^{1}$ Departamento de Astronomia, Instituto de Física, Universidade Federal do Rio Grande do Sul, Av. Bento Gonçalves 9500, \\ CP 15051, 91501-970 Porto Alegre, RS, Brasil \\ 2 Department of Astronomy and McDonald Observatory, University of Texas at Austin, Austin, Texas 78712-1083, USA \\ e-mail: fritz@astro.as.utexas.edu
}

Received 16 April 2003 / Accepted 29 July 2003

\begin{abstract}
We present low signal-to-noise spectroscopy and surface photometry of two small galaxies, detected in the field of the nearby asymmetric barred spiral NGC 7479, near a prolongation of the western arm that forms a faint tail in the disc, and selected as possible companions of this galaxy. The analysis was based on data obtained with the Prime Focus Camera (PFC) at the $0.8 \mathrm{~m}$ telescope of McDonald Observatory, and the Low Resolution Spectroscope (LRS) at the $9.2 \mathrm{~m}$ Hobby-Eberly Telescope (HET). We determined radial velocities using cross correlation with 15 different templates, and derived integrated magnitudes, colors and brightness profiles for each.

The derived radial velocities are around $35500 \mathrm{~km} \mathrm{~s}^{-1}$ for one galaxy and $38000 \mathrm{~km} \mathrm{~s}^{-1}$ for the other, which clearly indicates that both are far more distant than the nearby NGC 7479. The absolute magnitudes and diameters of these two background galaxies are typical of normal galaxies, and their colors are similar to other galaxies with similar redshifs. NGC 7479 continues to exist in isolation with no detected companion.
\end{abstract}

Key words. galaxies: individual: NGC 7479 - galaxies: photometry

\section{Introduction}

NGC 7479 is a grand-design spiral galaxy, classified as SB(s)c in RC3 (de Vaucouleurs et al. 1991), that has been extensively studied for its impressive features. It has an unusually strong bar, with dust lanes associated with molecular gas, detected in $\mathrm{CO}$, and regions of intense star formation (e.g. Benedict 1982; Quillen et al. 1995; Sempere et al. 1994; Martin \& Friedli 1997; Rozas et al. 1999; Aguerri et al. 2000. These last authors cataloged more than 1000 HII regions in the galaxy, including bar and disc. The spiral structure has as main components two spiral arms strongly asymmetric (Martin \& Friedli 1997), that apparently are leading (Beckman \& Cepa 1990; Puerari \& Dottori 1997), and present strong 21-cm line emission (Laine \& Gottesman 1998). The small and bright nucleus is classified as LINER by Keel (1983) and as Seyfert 1.9 by Ho et al. (1997). It presents a circumnuclear disc detected in CO (Sempere et al. 1994; Laine et al. 1999) and in HI (Laine \& Gottesman 1998). The infrared colors of the galaxy are typical of galaxies having bursts of star formation.

The overall morphology of the galaxy seems to be an excellent example of an interacting system, but it has no close companion, leading some authors (e.g. Quillen et al. 1995;

Send offprint requests to: M. F. Saraiva,

e-mail: fatima@if.ufrgs.br
Laine \& Gottesman 1998; Martin et al. 2000) to propose a model in which NGC 7479 would have suffered a recent merger with a low mass galaxy. This merger became even more likely after the simulations by Laine \& Heller (1999) in which they reproduce the appearance of the galaxy supposing it captured a small companion in a canibalization process that may not be completed yet.

In an attempt to find low surface brightness galaxies in the neighborhood of NGC 7479, we had several runs in 1995 with the Prime Focus Camera at the $0.8 \mathrm{~m}$ telescope of McDonald Observatory to obtain long exposure, wide field images of the galaxy. We found no companion. In a recent re-examination of the those images, we identified some features that might had added new evidence for the merger hypothesis. These images, after a very precise sky subtraction, reveal a prolongation of the western arm beyond the disc edge that forms a faint tail, not shown in any previous image of NGC 7479. Close to the tail, there are two very small faint galaxies. These features are shown in Fig. 1.

If these galaxies were at the same distance as NGC 7479, they could be tidal dwarf galaxies, a type of object that may be produced during galactic encounters (Duc \& Mirabel 1998).

This possibility prompted us to ask for time at the HET telescope to obtain spectra of the galaxies to measure their radial velocities. This would allow us to determine if they are dwarf 


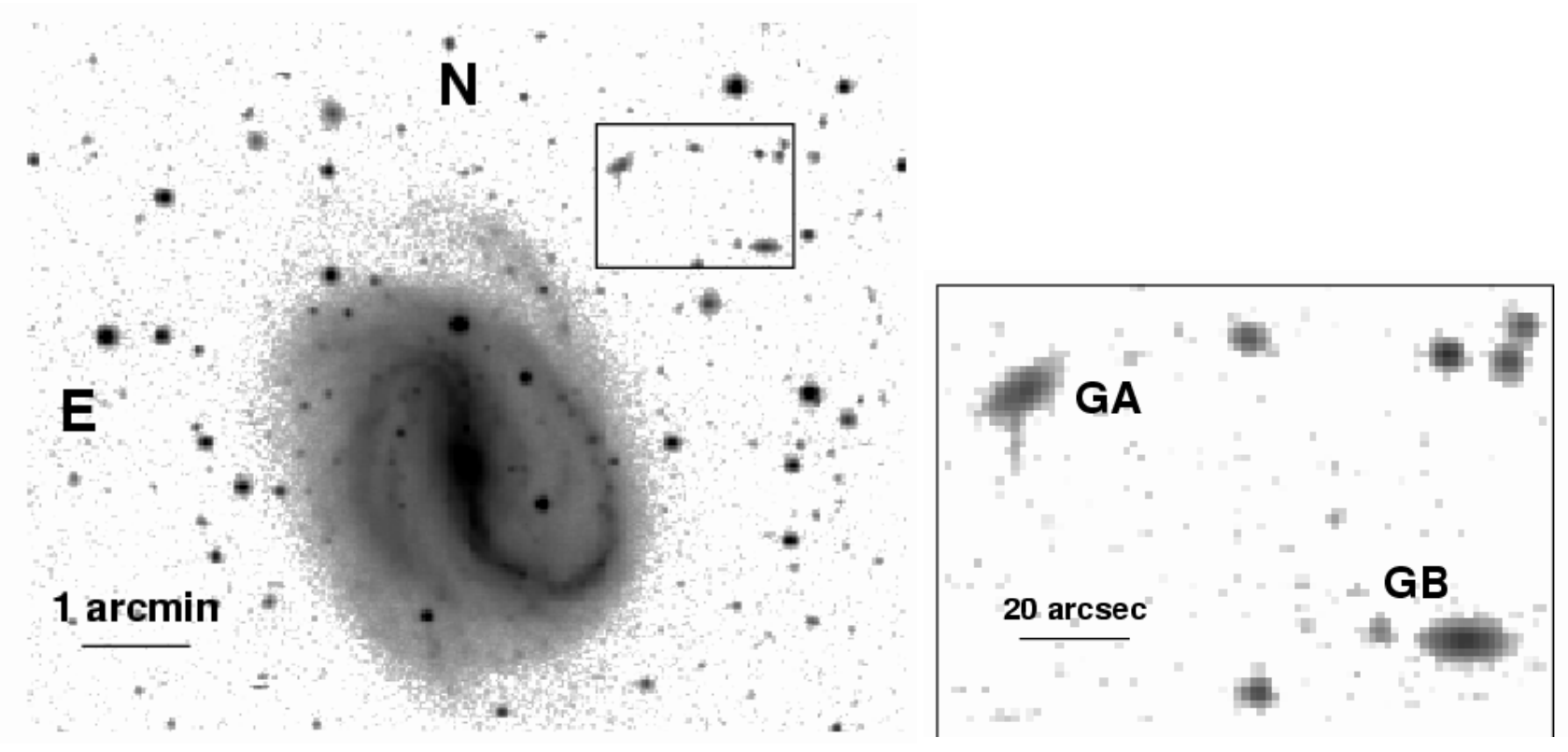

Fig. 1. The left panel shows NGC 7479 and, in the marked region, the two faint galaxies, that we will call NGC 7479-GA and NGC 7479GB, or GA and GB for short. This region appears zoomed in the right panel, showing GA at top left and GB at bottom right. The J2000 equatorial coordinates of the galaxies are $\alpha=23: 04: 50.8, \delta=12: 22: 08.4$, and $\alpha=23: 04: 45.3, \delta=12: 21: 24.6$, for GA and GB respectively. The 2MASS Extended Source Images (NASA/IPACInfrared Science Arquive (IRSA), has recently released observational results on GB: its identification is 2MASXJ23044541+1221233, and its total magnitudes are $J=15.259 \pm 0.230, H=14.384 \pm 0.198$, and $K_{\mathrm{s}}=13.744 \pm 0.258(\mathrm{NED})$.

companions of NGC 7479 or normal, more distant galaxies. In this paper we present those observations, in Sect. 2, the results from spectroscopy, in Sect. 3, and from surface photometry, in Sect. 4. In Sect. 5 we summarize our results.

\section{Observations and data reductions}

\subsection{Photometric data}

The photometric data were obtained at McDonald Observatory, using the 0.8-m telescope with the Prime Focus Camera (PFC). The detector was a $2048 \times 2048 \mathrm{CCD}$ with pixel size of $15 \mu$, corresponding to a plate scale of $1.35 \operatorname{arcsec}$ pix $^{-1}$. The field of view was $46 \times 46 \square^{\prime}$. The filters used were $B, V, R$ and $I$. Multiple exposures of standard stars from Landolt (1992) were also taken for the photometric calibration.

The images were corrected for bias and flat-field using standard IRAF routines. After the correction by atmosferic extinction the multiple exposures in each filter have been aligned and combined. The alignments were correct within 0.25 pixel in average. The sky background of each final broad-band image has been determined with the IRAF routine imsurfit, by fitting a 2nd order polynomial to the frame regions around the galaxy, after cleaning all the stars on them. The calculated sky values and their uncertainties are shown in Table 1.

The data was calibrated to the Johnson-Kron-Cousins system using observations of standard stars in the regions SA92 and SA97 of Landolt (1992). The transformation equations are given in another paper (Saraiva \& Benedict 2003, in preparation).
Table 1. Photometric observations.

\begin{tabular}{lccc}
\hline \hline Filter & $\begin{array}{c}F W H M \\
\text { arcsec }\end{array}$ & $\begin{array}{c}\text { Exposure time } \\
\mathrm{s}\end{array}$ & $\begin{array}{c}\text { Sky brightness } \\
\mu\end{array}$ \\
\hline$B$ & 3.0 & $3 \times 600$ & $22.34 \pm 0.027$ \\
$V$ & 2.7 & $2 \times 600$ & $21.39 \pm 0.021$ \\
$R$ & 2.7 & $3 \times 300$ & $20.75 \pm 0.011$ \\
$I$ & 3.1 & $3 \times 600$ & $19.37 \pm 0.012$ \\
\hline
\end{tabular}

Table 2. Spectroscopic observations

\begin{tabular}{lccc}
\hline \hline $\begin{array}{l}\text { Slit width } \\
\operatorname{arcsec}\end{array}$ & $\begin{array}{c}\text { Exposure } \\
\min \end{array}$ & $\begin{array}{c}\text { Spectral range } \\
\AA\end{array}$ & $\begin{array}{c}\text { Resolution } \\
\AA\end{array}$ \\
\hline 2 & $2 \times 10$ & $4300-7300$ & 4 \\
\hline
\end{tabular}

\subsection{Spectroscopic data}

The spectroscopic data were obtained with the Low Resolution Spectrograph (LRS) at the $9.2 \mathrm{~m}$ Hobby-Eberly Telescope (HET). The detector is a $1568 \times 512 \mathrm{CCD}$ binned $2 \times 2$, corresponding to a plate scale of $0.47 \mathrm{arcsec}_{\mathrm{pix}}{ }^{-1}$. We used a grating of $600 \mathrm{l} / \mathrm{mm}$, a slit width of 2 arcsec, and the filter GG385. The obtained resolution with this setup is $4 \AA$, and the spectral coverage is 4300-7300 $\AA$. The slit has total length of 4 arcmin and was properly oriented to include both galaxies. Two exposures of 10 min each were taken.

The data reduction, consisting of bias subtraction, flatfielding, sky subtraction, and wavelength calibration was 

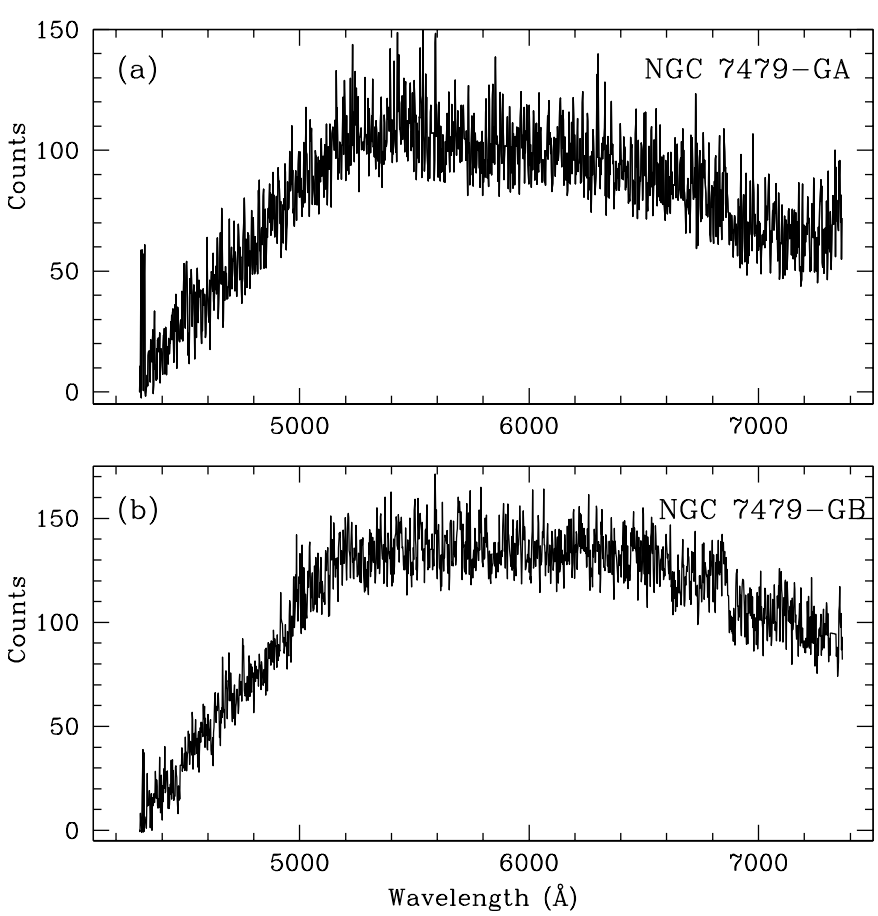

Fig. 2. a) Spectrum of NGC 7479-GA. The ordinate is the number of counts per pixel. b) Spectrum of NGC 7479-GB.

carried out using standard IRAF $^{1}$ routines. The cosmic rays were eliminated using the IRAF task cosmic ray prior to the spectra extraction, and the remaining ones have been eliminated by interpolation. The extraction window for each galaxy has been chosen to include the total galaxy appearing in the slit. The combination of the spectra was made after the extractions. The signal-to-noise after the combination was 8.5 for the galaxy A and 13.5 for the galaxy B, in the range 6000-6500. Figure 2 shows the spectra.

\section{Spectroscopy}

\subsection{Cross-correlation}

For determining the radial velocities we used the IRAF task fxcor, that applies the Fourier Cross Correlation technique, as described in Tonry \& Davis (1979 - hereafter TD79). Basically, the galaxy spectrum is correlated against the template spectrum and the resultant peak is fit by a smooth symmetric function.

Although usually stellar spectra are used as templates, in the case of poor signal-to-noise data, which is our case, galaxy spectra are more appropriate (TD79). We used three sets of galaxy templates: the first one is composed by the spectra of three elliptical galaxies (NGC 6851, NGC 6958 and ESO 18554) and one SO galaxy (IC 4214), taken at the Laboratório Nacional de Astrofísica, Brazil (LNA). Initially we tried to include in this set the spectra of two spiral galaxies (NGC 5757 and NGC 7412) but the correlation with these galaxies was very

${ }^{1}$ IRAF - Image Reduction and Analysis Facility - is distributed by the National Optical Astronomy Observatories, which is operated by the Association of Research in Astronomy, Inc., under cooperative agreement with the National Science Foundation, USA. poor, so we dropped them. The four template spectra used have good signal-to-noise, are normalized and shifted to zero redshift. Details on their characteristics are given in Saraiva et al. (2001).

The second set is composed by Las Campanas spectra of the elliptical galaxies NGC 1700, NGC 1426, and NGC 1407, plus a synthetic galaxy spectrum. These spectra have high signal-to-noise and are not shifted to zero redshift, with the obvious exception of the synthetic one.

The third set is composed by galaxy templates built from star clusters integrated spectra (Bica 1988).

Due to the very poor signal-to-noise of our object spectra, the true peak of the correlation was not easily recognized. In some cases there were other peaks of similar height. As recommended by TD79 we chose as main peak the one that showed up best (highest amplitude) in all the correlations.

\subsection{Radial velocities}

Tables 3, 4 and 5 summarize the correlation results for GA and GB. The parameters listed are the height of the chosen peak (Height), the ratio of the chosen peak to the average peak of the cross correlation function $(R)$, the $F W H M$ of the peak (Width), the velocity computed from the shift (Relative velocity), and the resulting velocity (Observed velocity). The two last columns have the same values if the template spectra have zero redshift, which is the case of first and third set.

Figures 3 and 4 show the cross-correlation of NGC 7479GA and NGC 7479-GB with NGC 6851. The cross-correlation functions of the two galaxies with the other templates have nearly the same shape and values.

Although in most cases the $R$ value is very small, and the computed velocity has a large internal uncertainty, all the 15 correlations computed for each galaxy gave practically the same result, which make us confident it is correct. The mean resulting radial velocities are $(35926 \pm 413) \mathrm{km} \mathrm{s}^{-1}$ for NGC 7479-GA and $(38009 \pm 83) \mathrm{km} \mathrm{s}^{-1}$ for NGC 7479-GB.

\section{Surface photometry}

In spite of the very low signal-to-noise ratio of the galaxies, we were able to calculate some photometric parameters, specifically diameters, ellipticities, position angles, integrated magnitudes and colors, and surface brightness profiles along the major axis.

\subsection{Ellipse fits}

In order to be able to determine geometric parameters using the task ellipse in the STSDAS package for IRAF, we artificially increased the resolution of each galaxy image, to have a number of points large enough to allow the calculation of the ellipses. We have done that using the IRAF task magnify, with a magnification factor of 10 . We used these magnified images only for the purpose of obtaining the isophote parameters, all the remaining analyses were based on the original images.

Figure 5 shows the variation of the surface brightness $(\mu)$, ellipticity $(\epsilon)$, position angle of the major-axis (PA), and the 
Table 3. Correlation results for the first set.

\begin{tabular}{lcccccc}
\hline \hline Galaxy & Template & Height & $R$ & Width & $\begin{array}{c}\text { Relative } \\
\text { velocity } \\
\mathrm{km} \mathrm{s}^{-1}\end{array}$ & $\begin{array}{c}\text { Observed } \\
\text { velocity } \\
\mathrm{km} \mathrm{s}^{-1}\end{array}$ \\
\hline N7479-GA & N 6851 & 0.123 & 3.428 & 3011 & 35957 & $35957 \pm 534$ \\
N7479-GA & N 6958 & 0.112 & 2.808 & 2922 & 35842 & $35842 \pm 602$ \\
N 7479-GA & I 4214 & 0.104 & 2.945 & 1371 & 36034 & $36034 \pm 664$ \\
N7479-GA & E 185-G54 & 0.114 & 2.847 & 4788 & 36112 & $36112 \pm 977$ \\
N7479-GB & N 6851 & 0.235 & 2.721 & 2977 & 37920 & $37920 \pm 628$ \\
N7479-GB & N 6958 & 0.227 & 2.939 & 2540 & 38100 & $38100 \pm 506$ \\
N7479-GB & I 4214 & 0.210 & 2.925 & 2319 & 38040 & $38040 \pm 464$ \\
N7479-GB & E 185-G54 & 0.196 & 2.33 & 2712 & 38232 & $38232 \pm 638$ \\
\hline
\end{tabular}

Table 4. Correlation results for the second set.

\begin{tabular}{lcccccc}
\hline \hline Galaxy & Template & Height & $R$ & Width & $\begin{array}{c}\text { Relative } \\
\text { velocity } \\
\mathrm{km} \mathrm{s}^{-1}\end{array}$ & $\begin{array}{c}\text { Observed } \\
\text { velocity } \\
\mathrm{km} \mathrm{s}^{-1}\end{array}$ \\
\hline N 7479-GA & N 1407 & 0.220 & 3.46 & 1902 & 34456 & $35896 \pm 334$ \\
N 7479-GA & N 1426 & 0.139 & 3.11 & 844 & 33151 & $34798 \pm 161$ \\
N 7479-GA & N 1700 & 0.152 & 2.95 & 1051 & 30526 & $34888 \pm 300$ \\
N 7479-GA & synth & 0.157 & 3.77 & 1254 & 34987 & $34987 \pm 206$ \\
N 7479-GB & N 1407 & 0.404 & 10.58 & 3020 & 36020 & $37986 \pm 331$ \\
N 7479-GB & N 1426 & 0.282 & 2.184 & 1289 & 36370 & $38031 \pm 169$ \\
N 7479-GB & N 1700 & 0.362 & 5.256 & 1973 & 33544 & $37925 \pm 212$ \\
N 7479-GB & synth & 0.28 & 5.18 & 1037 & 37965 & $37965 \pm 98$ \\
\hline
\end{tabular}

Table 5. Correlation results for the third set.

\begin{tabular}{lcccccc}
\hline \hline Galaxy & Template & Height & $R$ & Width & $\begin{array}{c}\text { Relative } \\
\text { velocity } \\
\mathrm{km} \mathrm{s}^{-1}\end{array}$ & $\begin{array}{c}\text { Observed } \\
\text { velocity } \\
\mathrm{km} \mathrm{s}^{-1}\end{array}$ \\
\hline N7479-GA & E1 & 0.148 & 3.217 & 2840 & 35549 & $35549 \pm 529$ \\
N7479-GA & E2 & 0.154 & 3.132 & 3030 & 35425 & $35425 \pm 576$ \\
N7479-GA & E3 & 0.156 & 3.159 & 3561 & 35623 & $35623 \pm 672$ \\
N7479-GA & E4 & 0.162 & 4.053 & 1757 & 35187 & $35187 \pm 273$ \\
N7479-GA & E5 & 0.168 & 3.665 & 3592 & 35487 & $35487 \pm 604$ \\
N7479-GA & E6 & 0.184 & 3.751 & 3705 & 36484 & $36484 \pm 612$ \\
N7479-GA & E7 & 0.168 & 3.756 & 2938 & 35429 & $35429 \pm 485$ \\
N7479-GB & E1 & 0.216 & 3.238 & 2086 & 37982 & $37982 \pm 386$ \\
N7479-GB & E2 & 0.263 & 3.103 & 2130 & 37961 & $37961 \pm 407$ \\
N7479-GB & E3 & 0.278 & 4.856 & 2078 & 38071 & $38071 \pm 278$ \\
N7479-GB & E4 & 0.184 & 2.917 & 1279 & 37927 & $37927 \pm 257$ \\
N7479-GB & E5 & 0.244 & 2.995 & 2115 & 37940 & $37940 \pm 415$ \\
N7479-GB & E6 & 0.340 & 4.956 & 2224 & 38025 & $38025 \pm 293$ \\
N7479-GB & E7 & 0.277 & 3.515 & 1907 & 38044 & $38044 \pm 331$ \\
\hline
\end{tabular}

$\cos 4 \theta$ term, with the $1 / 4$ power of the ellipse semi-major axis $\left(a^{1 / 4}\right)$.

These diagrams are significant only for $a^{1 / 4} \geq 1.1(a \geq$ $1.5)$ since the real resolution is $1.35^{\prime \prime}$. We can see that, in the significant range, GB presents smooth and coherent variations in the four filters, while in GA the isophotes are clearly more disturbed, with parameters varying differently in the different filters. Particularly, the position angle of the inner isophotes in the $V$ filter (triangles) presents a shift of $10^{\circ}$ with respect to the other filters.

We adopted as PA and $\epsilon$ of the galaxies the values obtained from the average of the fittings to the outermost isophotes in the four filters. The values for $\mathrm{GA}$ are $\mathrm{PA}=119^{\circ}$ and $\epsilon=0.44$, and for $\mathrm{GB}$ are $\mathrm{PA}=87^{\circ}$ and $\epsilon=0.52$. The shape parameter, $B 4$, indicates that GB has disky isophotes, but does not allow us to take any conclusion for GA.

\subsection{Luminosity profiles}

The surface brightness, shown in Fig. 6, span a range of three magnitudes. The observed central surface brightness is $\mu_{B} \simeq$ 23.3 for both galaxies. Their major axes at $\mu_{B}=26$, measured in these profiles, are 14" (GA) and 18" (GB). The shape of the color profiles are almost totally masked by the errorbars, but 


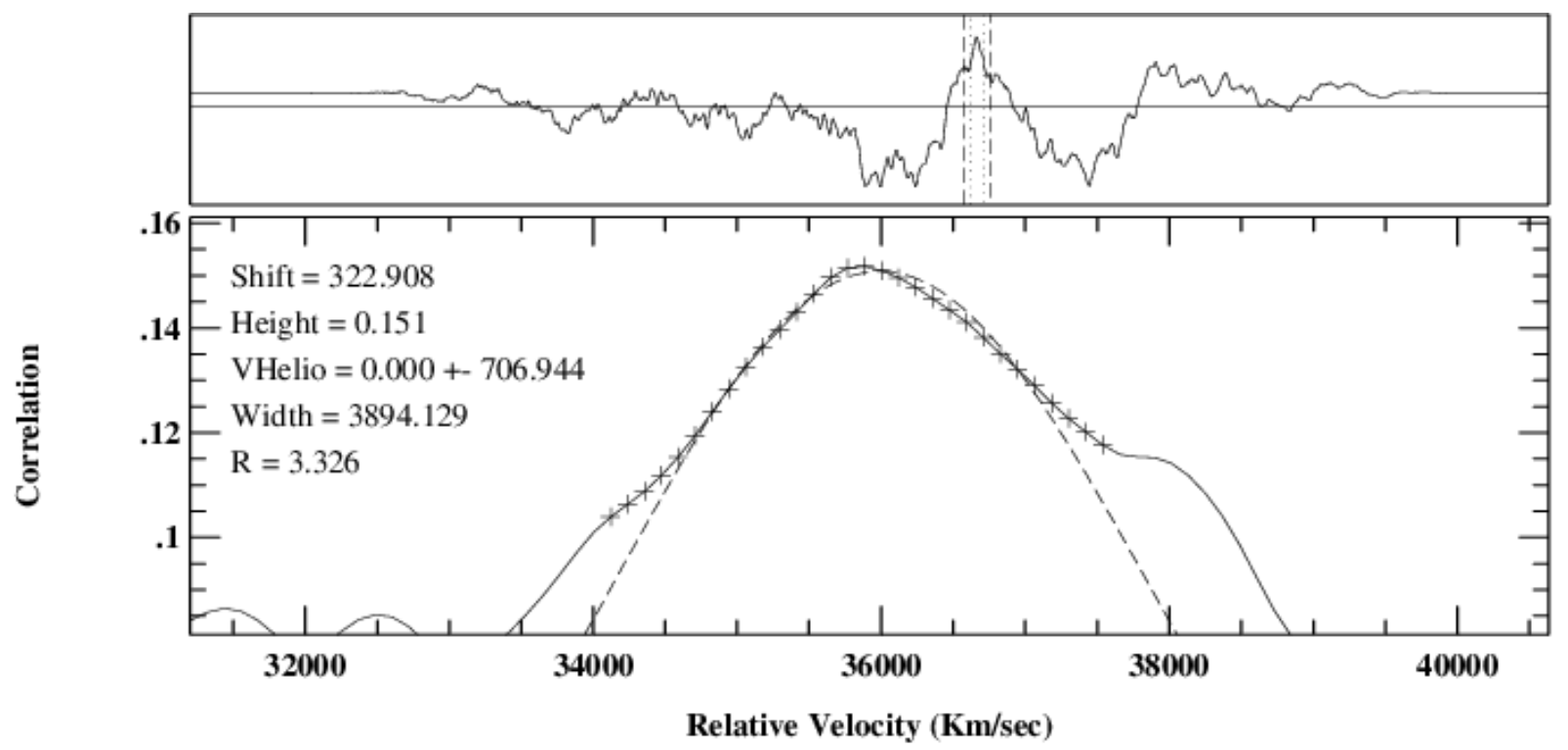

Fig. 3. The top panel is the cross-correlation function of NGC 7479-GA with NGC 6851, with the main peak between dashed lines. The bottom panel shows the fitting of the main peak.

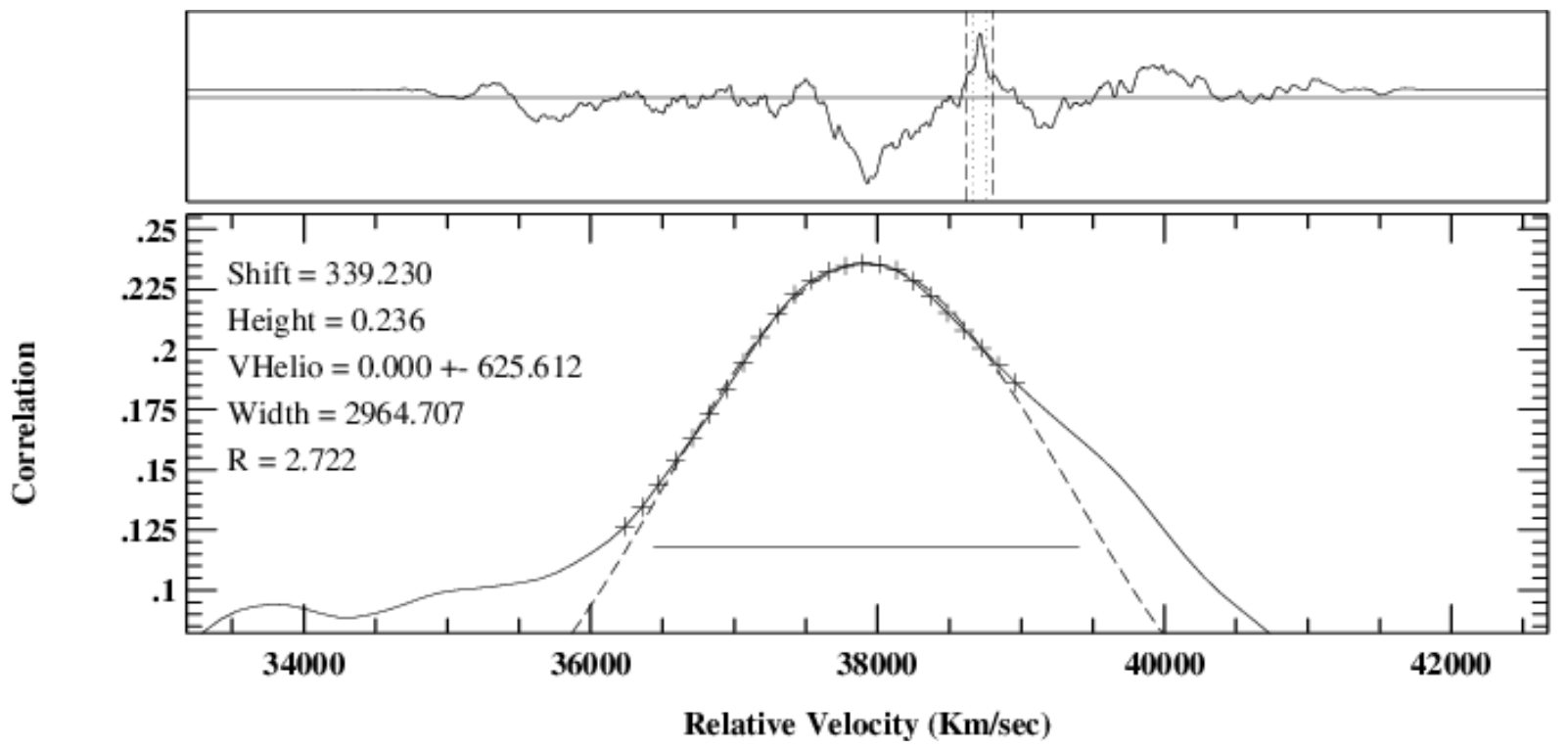

Fig. 4. Cross correlation of NGC 7479-GB with NGC 6851.

they still allow to notice that GB has a smooth surface brightness distribution, while GA seems to have some inner feature that disturbs its brightness distribution and causes the asymmetry in their color profiles. This might be an indication that the morphological type of GA is later than GB.

\subsection{Integrated magnitudes}

The integrated magnitudes were calculated within apertures of 10.8 and 13.5 arcsec for both galaxies, and the corresponding colors were obtained.

These apertures were selected attempting to include the maximum flux with the minimum noise, for which we used the criterium that the aperture should be the closest one to twice the $F W H M$ of the corresponding galaxy.
These parameters were corrected for Galactic extinction, adopting the values for NGC 7479 from Schlegel et al. (1998), from NED, and for redshift (k-correction), interpolating from tables provided by Poggianti (1997) for types E, Sa and Sc (Brown et al. 2001).

The adopted Galactic extinction values are $A_{B}=0.482$, $A_{V}=0.370, A_{R}=0.299$, and $A_{I}=0.217$, and the interpolated $\mathrm{k}$-correction values for $z=0.12$ are $k_{B}=0.52, k_{V}=0.173$, $k_{R}=0.10$, and $k_{I}=0.037$. These $k_{B}$ and $k_{V}$ values are intermediate between those given in Whitford (1971) and to those obtained following RC3 (de Vaucouleurs et al. 1991) for morphological type $\mathrm{Sa}(T=1)$.

The integrated magnitudes and colors of the two galaxies are shown in Table 6 . The quoted errors refer to the internal uncertainty in the measurement of the magnitude, due to uncertainties in the sky subtraction, and in the case of colors were 

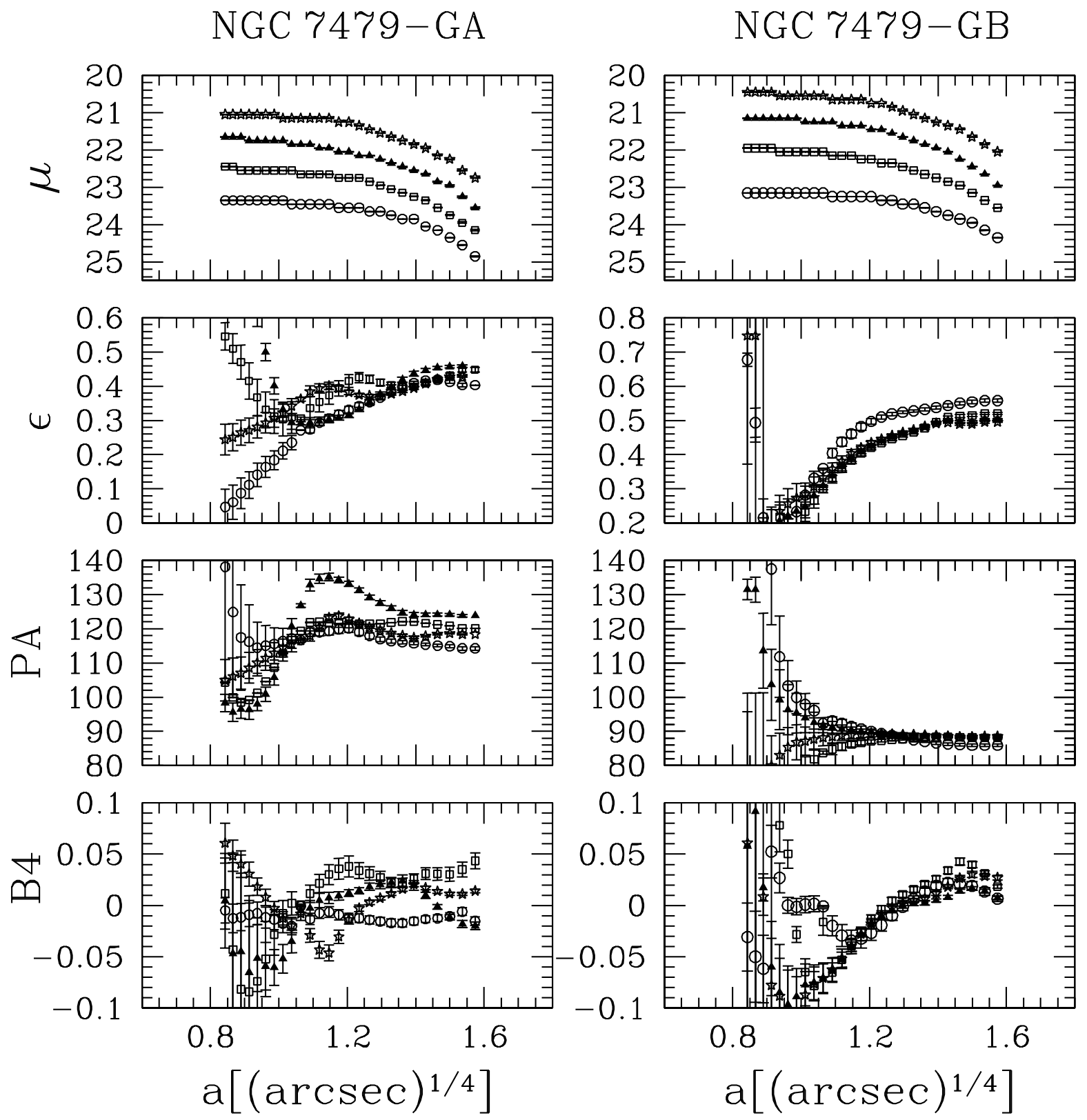

Fig. 5. Isophotal parameters surface brightness $(\mu)$, ellipticity $(\epsilon)$, position angle (PA), and $\cos 4 \theta$ Fourier term (B4), as a function of the semi-major axis $\left(a^{1 / 4}\right)$. The symbols circles, squares, triangles, and stars, represent the filters $B, V, R$, and $I$, respectively.

calculated as the sum in quadrature of the uncertainty in each magnitude. The integrated parameters show that GB is slightly brighter and significantly redder than GA, mainly in $B-V$, what is in agreement with the local color profiles.

Comparing the corrected colors with the mean BVRI colors of nearby galaxies, (e.g. Buta \& Willians 1995; Marakova 1999) both GA and GB have nontypical colors, mainly GA, that is both blue in $B-V$ and red in $V-I$. Probably a best comparison sample is provided by Bershady et al. (1994), who present $U J F N K$ colors of galaxies at $z \leq 0.25$, with median $z=0.12$. Since these authors made no correction for reddening or redshift, we used our uncorrected $B V R I$ colors, after transformation to $J F N$ colors through the equations given in Majewski (1992), for comparison purposes The transformed colors are $(J-F)=0.75-0.78,(F-N)=0.87-0.90$ for GA, and $(J-F)=1.06-1.16,(F-N)=0.95-1.04$ for GB, which are within the $(J-F)$ vs. $(F-N)$ distribution in Fig. 17c of Bershady et al. (1994). It is not clear if this difference between the mean colors of galaxies at $z \simeq 0.12$ and those nearby is real or arises from the lack of a precise correction for Galactic absorption and redshift.

\section{Summary}

With low resolution spectra and CCD images we determined radial velocities and photometric parameters of two small galaxies (GA and GB) in the field of NGC 7479. The radial velocities were calculated using the cross-correlation technique and three sets of templates, in a total of 15 measurements for each galaxy; the mean values were $(35513 \pm 413) \mathrm{km} \mathrm{s}^{-1}$ for NGC 7479-GA and $(38009 \pm 83) \mathrm{km} \mathrm{s}^{-1}$ for NGC 7479-GB 

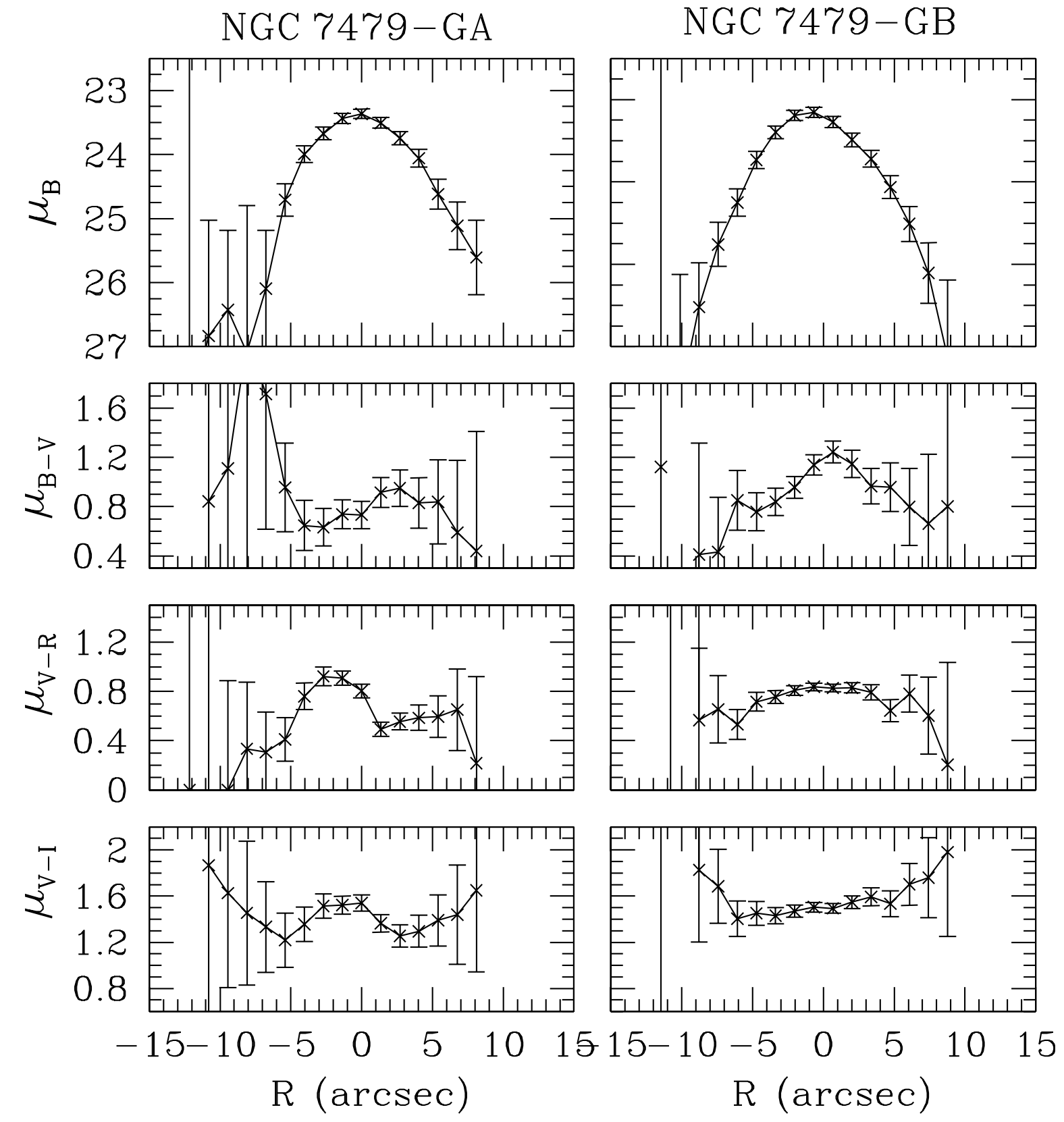

Fig. 6. Surface brightness profiles along the major axis of the galaxies A and B. The position angles are $115^{\circ}$ for GA and $87^{\circ}$ for GB. The error bars take into account the uncertainty in the sky subtraction.

Table 6. Integrated magnitudes and colors.

\begin{tabular}{lcccc}
\hline \hline Parameter & \multicolumn{2}{c}{ N7479-GA } & \multicolumn{2}{c}{ N7479-GB } \\
aperture & $10.8^{\prime \prime}$ & $13.5^{\prime \prime}$ & $10.8^{\prime \prime}$ & $13.5^{\prime \prime}$ \\
\hline$B$ & $19.27 \pm 0.06$ & $19.12 \pm 0.06$ & $19.20 \pm 0.06$ & $19.03 \pm 0.06$ \\
$V$ & $18.63 \pm 0.05$ & $18.49 \pm 0.05$ & $18.22 \pm 0.04$ & $18.10 \pm 0.06$ \\
$R$ & $17.93 \pm 0.04$ & $17.82 \pm 0.04$ & $17.44 \pm 0.03$ & $17.32 \pm 0.03$ \\
$I$ & $17.16 \pm 0.02$ & $17.01 \pm 0.03$ & $16.67 \pm 0.02$ & $16.52 \pm 0.02$ \\
$(B-V)$ & $0.64 \pm 0.08$ & $0.63 \pm 0.08$ & $0.98 \pm 0.07$ & $0.93 \pm 0.08$ \\
$(V-R)$ & $0.70 \pm 0.06$ & $0.67 \pm 0.06$ & $0.77 \pm 0.05$ & $0.78 \pm 0.07$ \\
$(V-I)$ & $1.47 \pm 0.05$ & $1.48 \pm 0.06$ & $1.55 \pm 0.04$ & $1.58 \pm 0.06$ \\
$B^{0}$ & 18.33 & 18.18 & 18.24 & 18.07 \\
$(B-V)^{0}$ & 0.19 & 0.18 & 0.53 & 0.48 \\
$(V-R)^{0}$ & 0.54 & 0.51 & 0.63 & 0.64 \\
$(V-I)^{0}$ & 1.16 & 1.17 & 1.28 & 1.31 \\
\hline
\end{tabular}


Despite the large uncertainty, these values indicate clearly that the two faint galaxies are much farther away than NGC 7479, which heliocentric radial velocity is $2381 \mathrm{~km} \mathrm{~s}^{-1}$ (NED NASA/IPAC Extractragalactic Database).

The photometric analysis yielded isophote parameters, luminosity and color profiles, and integrated magnitudes and colors. Our results suggest that the galaxies have similar sizes and brightnesses, but different morphological types, since GB is significantly smoother and redder than GA.

Assuming a Hubble constant of $H_{0}=75 \mathrm{~km} \mathrm{~s}^{-1} \mathrm{Mpc}^{-1}$, the derived distances of the galaxies GA and GB are $480 \mathrm{Mpc}$ and $500 \mathrm{Mpc}$ respectively, corresponding to a mean scale $1^{\prime \prime}=2.35 \mathrm{kpc}$ and mean distance modulus $(m-M)=38.44$. Using the integrated magnitudes determined in Sect. 4, corrected for redshift, both galaxies have absolute magnitudes around $M_{B}^{\mathrm{o}}=-19.7$, and major diameters around $30 \mathrm{kpc}$, which are typical of normal nearby galaxies. Their colors are common among galaxies with similar redshift, but seem to be rare among nearby ones.

Acknowledgements. We deeply thank Dr. Claudia Mendes de Oliveira for clarifying discussion on the subject, as well as for kindly giving us four spectra prior to publication. We thank Dr. Charles Bonatto for careful reading of the manuscript, and the referee, Dr. J. A. Aguerri, for suggestions that really help to improve paper. This research has made use of NASA's Astrophysics Data System Abstract Service (ADS), and the NASA/IPAC Extragalactic Database (NED) which is operated by the Jet Propulsion Laboratory, California Institute of Technology, under contract with the National Aeronautics and Space Administration.

\section{References}

Aguerri, J. A. L., Varela, A. M., Prieto, M., \& Munoz-Tunon, C. 2000, AJ, 119, 1638

Benedict, G. F. 1982, AJ, 87, 76

Beckman, J. E., \& Cepa, J. 1990, A\&A, 229, 37

Bershady, M. A., Hereld, M., Kron., R., et al. 1994, AJ, 108, 870

Bica, E. 1988, A\&A, 195, 76

Brown, W. R., Geller, M. J., Fabricant, D. G., \& Kurtz, M. J. 2001, AJ, 122, 704

Buta, R., \& Williams K. L. 1995, AJ, 109, 543

de Vaucouleurs, G., de Vaucouleurs, A., Corwin, H., et al. 1991, The Third Reference Catalogue of Bright Galaxies, (New York: Springer Verlag) (RC3)

Duc, P.-A., \& Mirabel, I. F. 1998, A\&A, 333, 813

Ho, L., Filippenko, A., \& Wallace, L. W. 1997, ApJS, 112, 315

Laine, S., \& Gottesman, S. T. 1998, MNRAS, 297, 104

Laine, S., \& Heller, C. H. 1999, MNRAS, 308, 557

Keel, W. C. 1983, 269, 466

Laine, S., Kenney, J. D. P., Yun, M. S., et al. 1999, ApJ, 511,709

Landolt, A. U. 1992, AJ, 104, 340

Makarova, L. 1999, A\&AS, 139, 491

Majewski, S. R. 1992, ApJS, 78, 87

Martin, P., \& Friedli, P., A\&A, 326, 449

Martin, P., Lelievre, M., \& Roy, J.-R. 2000, ApJ, 538, 141

Poggianti, B. M. 1997, A\&A, 122, 399

Puerari, I., \& Dottori, H. 1997, ApJ, 476, L73.

Quillen, A. C., Frogel, J. A., Kenney, et al. 1995, ApJ, 441, 549

Rozas, M., Zurita, A., Heller, C. H., et al. 1999, A\&AS, 135,145

Saraiva, M. F., Bica, E., \& Pastoriza, M. G., et al. 2001, A\&A, 376, 43

Schlegel, D. S., Finkbeiner, D. P., \& Davis, M. 1998, ApJ, 500, 525

Sempere, M. J., Combes, F., \& Casoli, F. 1994, A\&A, 299, 371

Tonry, J., \& Davis, M. 1979, AJ, 84, 1511

Whitford, A. E. 1971, ApJ, 169, 215 